\title{
A aplicação do método SAMCurve para a avaliação da experiência de usuário no uso de sistemas web
}

\author{
Elvis Leite da Silva ${ }^{1}$, Rodrigo Campos Bortoletto ${ }^{1}$ \\ ${ }^{1}$ Instituto Federal de São Paulo (IFSP) - Guarulhos, SP - Brasil \\ elvis.leitedaluno.ifsp.edu.br, bortolettodifsp.edu.br
}

\begin{abstract}
The spread of the use of web systems, and the subsequent expansion of the use of computers and other digital devices, has generated major problems related to the experience of using corporate web systems, these can be positive or negative, but as experiences and emotions are not events singular, but rather continuous events that occur over time. In this longitudinal study, the UX SAMCurve evaluation method was used, recently developed to evaluate a user experience, focusing on hedonic categories over the time of use of a web system.
\end{abstract}

Resumo. A disseminação do uso de sistemas web, e a subsequente expansão do uso de computadores e outros dispositivos digitais, gerou grandes problemas relacionados com a experiência de uso de sistemas web corporativos, essas podem ser positivas ou negativas, porém as experiências e emoções não são eventos singulares, mas sim eventos contínuos que ocorrem ao longo do tempo. Neste estudo longitudinal foi utilizado o método de avaliação UX SAMCurve, desenvolvido recentemente para avaliar a experiência do usuário, com foco nas qualidades hedônicas ao longo do tempo de utilização de um sistema web.

\section{Introdução}

O contexto atual da pandemia ocasionada pelo novo coronavírus (SARS-CoV-2) tornouse um dos maiores desafios da humanidade neste século, foram afetadas direta e/ou indiretamente setores como saúde, educação e a economia da população mundial [Werneck and Carvalho 2020]. Uma das medidas recomendadas segundo o Conselho Nacional de Saúde para evitar a disseminação do vírus foi o distanciamento social e como consequência desse isolamento houve um aumento do uso de tecnologias digitais [BRASIL 2021], presumindo que a tecnologia afastou a necessidade de proximidade entre as pessoas [Cavalcanti 2020]. Neste sentido, segundo a pesquisa da Associação Brasileira de Comércio Eletrônico (ABComm) realizada entre $1^{\circ}$ de março e 6 de junho de 2020, levando em consideração apenas vendas online no Brasil indicou que 20,2 milhões de consumidores realizaram pela primeira vez uma compra pela internet [ABComm ], outra pesquisa feita em 2020 pela Associação Brasileira das Empresas de Software apontou que as soluções de Software como serviço (SaaS, do inglês System as a Service) tiveram um aumento de $28,5 \%$ [ABES ].

A disseminação do uso de sistemas web, e a subsequente utilização de computadores e outros dispositivos digitais, gerou grandes problemas relacionados com a experiência de uso desses sistemas nas corporações, porém a experiência de utilizar um sistema web normalmente pode ser positiva ou negativa, a experiência é considerada positiva quando o usuário consegue realizar a tarefa sem demora, frustração ou sem encontrar problemas 
[Teixeira 2014]. Diante desse cenário, o campo de Interação Humano Computador (IHC) abrange várias áreas científicas como a ciência da computação, ciência ergonômica, psicologia cognitiva e engenharia dos fatores humanos [Lazar et al. 2017]. Considerando os fatores humanos, a experiência do usuário (UX, acrônimo de User eXperience) é definida como um conceito de algo que abrange todos os aspectos da experiência que uma pessoa poderia ter ao interagir com um produto/serviço [Norman 2006]. As experiências e emoções não são eventos singulares, mas eventos contínuos, visto que a relação entre usuário e produto/serviço evolui ao longo do tempo [Forlizzi and Battarbee 2004, Kujala et al. 2011].

Diante desse contexto, este artigo tem como objetivo utilizar o método de avaliação UX SAMcurve para avaliar a experiência do usuário, com foco nas qualidades hedônicas, ao longo do tempo de utilização de um sistema web em um ambiente corporativo. Pretende-se verificar quais os resultados que este tipo de técnica produz e qual a percepção dos usuários ao utilizá-las para reportarem suas experiências com a aplicação, além disso, esse trabalho também traz como contribuição a continuidade dos estudos apresentados por [Alves 2020]. A sequência do texto está organizada da seguinte forma: na seção 2, apresenta-se o referencial metodológico com o método de avaliação de UX utilizado, na seção 3 a caracterização dos participantes e a descrição do sistema web testado. Na seção 4 são apresentados e analisados os resultados e por fim, na seção 5 são feitas as considerações finais.

\section{Método de avaliação}

O método SAMCurve é inspirado no artefato UX Curve, e conceitualmente baseado na escala gráfica (SAM, do inglês Self-Assessment Manikin) para avaliar os três estados afetivos: Satisfação, Motivação e Controle.O SAMCurve foi inicialmente idealizado para ser utilizado com papel e caneta, as curvas são desenhadas incluindo pontos que representam os estados afetivos de uma pessoa, durante um determinado período, enquanto realizam atividades específicas. O participante desenha uma ou mais curvas para descrever como a experiência sobre a utilização de um produto ou serviço mudou ao longo do tempo [Kujala et al. 2011, Bradley 1994].

A área de desenho da curva Figura 1 é formada por uma linha de tempo e uma linha horizontal que divide experiências positivas e negativas. Para fins de tabulação é adotado como critério de pontuação, uma escala de 1 a 5 pontos (da esquerda para a direita) considerando como dimensões: Satisfação, Motivação e Controle.

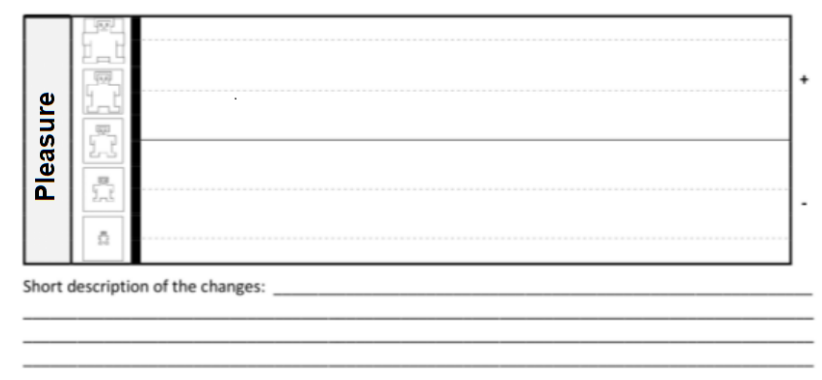

Figura 1. Modelo para a dimensão "Satisfação" do SAMCurve para preenchimento com papel e caneta [Alves 2020]. 
- Satisfação: o quão satisfeito o usuário ficou quando ele estava utilizando o artefato computacional;

- Motivação: o quão empolgado o usuário se sente durante o tempo em que ele utilizou o artefato computacional;

- Controle: qual o domínio do usuário sobre o artefato computacional.

\subsection{Participantes}

Foi considerado para essa pesquisa um grupo heterogêneo de funcionários da Empresa $\mathrm{X}$, uma empresa de transporte público de passageiros, situada na cidade de Guarulhos, São Paulo, onde foi desenvolvido e implantado o artefato computacional. O moderador foi o autor que não possui relação prévia com os participantes. A pesquisa foi conduzida com vinte funcionários voluntários, com níveis de escolaridade distintos e buscando obter diferentes perspectivas, sendo está uma amostra intencional que foi recrutada com diferenciação de idade entre 22 e 64 anos.

$\mathrm{O}$ experimento fez parte das atividades cotidianas dos funcionários, para a aceitação do estudo, os voluntários preencheram um Termo de Consentimento Livre e Esclarecido (TCLE) tomando ciência que os dados coletados teriam a finalidade única para o estudo, e que sua identidade seria preservada a todo momento e suas respostas seriam anônimas. O convite foi realizado por meio de e-mail corporativo da referida empresa, e a participação no estudo foi voluntária.

\subsection{Artefato Computacional}

Foi definido como artefato computacional o sistema web desenvolvido em php e bootstrap, este sistema trata-se de uma aplicação responsiva de Groupware, para gestão de frota, que visa interligar as diferentes áreas dentro da organização, para prover uma comunicação e execução de tarefas. O sistema controla o fluxo de veículos na portaria, permitindo que os usuários colaborem de forma síncrona e/ou assíncrona. Este sistema pode ser acessado por meio de um endereço web pelos navegadores tanto por meio de desktops quanto por meio smartphones, Figura 2.

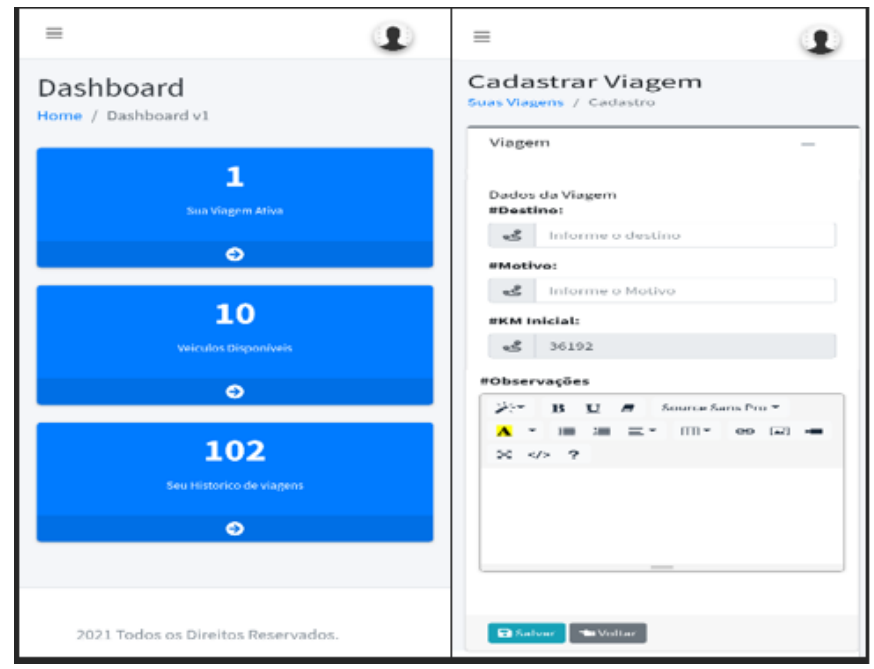

Figura 2. Telas do sistema para o acesso por meio de smartphones.

Fonte: Autores 
Características de utilização:

- $O$ usuário acessa o sistema via navegador;

- O usuário loga no sistema;

- $O$ usuário seleciona um veículo disponível;

- $O$ usuário registra a saída da viagem;

- $O$ usuário ao chegar do destino finaliza a viagem.

\subsection{Procedimentos de coleta de dados}

A avaliação foi conduzida com a aplicação de um experimento baseado na realização de uma tarefa cotidiana, a partir do uso de um sistema web. A amostra foi intencional, e ficou definido como ambiente de estudo um cenário real que permite avaliar as interações de modo mais natural, todos os participantes foram convidados a explorar o sistema por um período pré-determinado, sendo aplicada a avaliação uma vez por semana durante 6 semanas consecutivas, tendo inicio em 12/07/2021 e encerrado no dia 16/08/2021.

Antes do início da coleta de dados, o moderador apresentou os objetivos da pesquisa aos participantes e solicitou o preenchimento de um pré-cadastro por meio de formulário eletrônico, de modo a garantir o total caráter voluntário e anônimo da participação. Ao confirmar a participação na pesquisa, o participante recebia um link de acesso ao sistema web e outro link para um formulário eletrônico para o participante relatar suas experiências, optou-se por esse procedimento devido ao isolamento social decorrente da pandemia de Covid-19.

A tarefa dos participantes consistia na utilização do sistema, após a conclusão do registro de uma viagem completa, os participantes efetuaram a avaliação de experiência no formulário eletrônico. Nessa avaliação, o participante teve que escolher dentre cinco figuras, na escala de dimensões para Satisfação, Motivação e Controle do SAMCurve, de modo a indicar como ele estava se sentindo em relação a cada uma dessas dimensões naquele momento. No final de cada etapa de coleta, havia um espaço não obrigatório para que o participante descrevesse como ele avaliava a dimensão correspondente.

\section{Resultados}

Os dados foram analisados de acordo com a definição do método, considerando cada uma das dimensões em uma escala de cinco pontos, variando de um a cinco, no qual o valor um representa pouco controle, pouca satisfação ou pouca motivação, o valor três representa o estado neutro, e o valor cinco representa muita satisfação, muita motivação ou muito controle.

\subsection{Relação das menções dos participantes e suas implicações nas curvas}

Além de fornecerem dados para formar as curvas, os participantes descreveram os fatores que melhoraram sua experiência ou causaram deterioração. Razões hedônicas positivas foram associadas à melhoria das curvas e razões hedônicas negativas foram associadas a deterioração da curva. Para testar essa hipótese, comparou-s os resultados do questionário final de cada dimensão com os picos das curvas.

As perguntas abertas: "Como você avalia a motivação ao utilizar o sistema?", "Como você avalia a satisfação ao utilizar o sistema?" e "Como você avalia o domínio (controle) sobre a utilização do sistema?” foram feitas ao final de cada dimensão em cada 
um dos seis momentos de coleta de dados no experimento. Ao contrário das respostas objetivas, essas questões eram de preenchimento opcional, dessa forma, foram coletadas 202 respostas às questões ao longo do teste, sendo realizada uma análise na tentativa de relacionar as respostas com os momentos de alteração das curvas.

Para realização dessa análise, as respostas dos participantes foram codificadas em categorias pré-estabelecidas, as quais foram constituídas pelas três dimensões presentes no SAMCurve. O conjunto de respostas foi classificado por dois codificadores, que atribuíram para cada comentário uma medida de concordância. Para verificar a confiabilidade da codificação, utilizou-se o coeficiente de Cohen's Kappa [Lazar et al. 2017], que é baseada no número de respostas concordantes, ou seja, no número de casos cujo resultado é o mesmo entre os codificadores, o qual indica que um valor acima de $60 \%$ representa uma confiabilidade satisfatória. O coeficiente calculado para a codificação realizada foi de $61,8 \%$, a Figura 3 apresenta o gráfico dos 152 comentários feitos pelos participantes, divididos por dimensão, os quais tiveram concordância entre os codificadores.

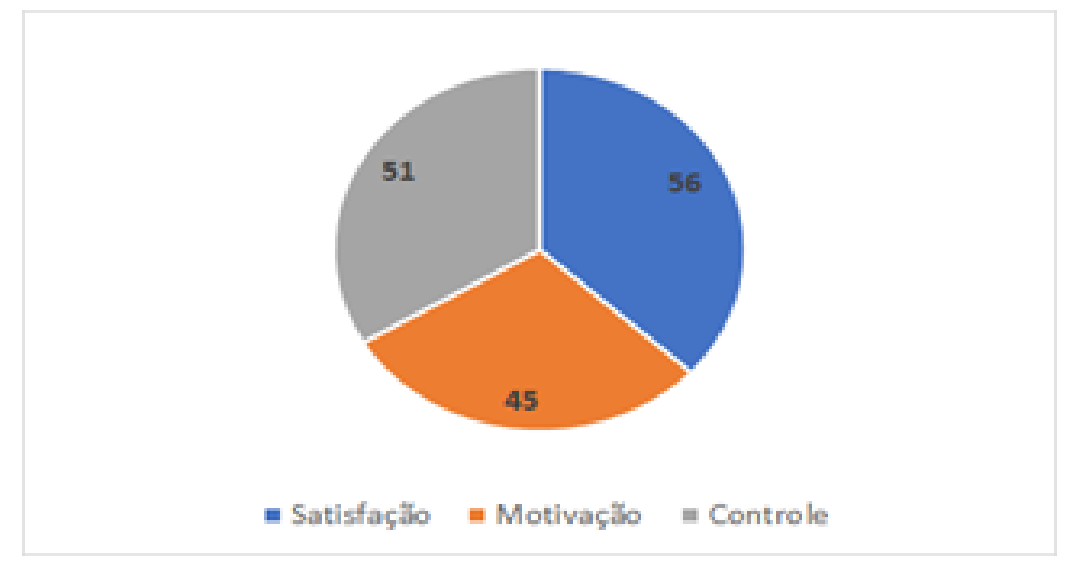

\section{Figura 3. Quantidade de respostas associadas às dimensões Satisfação, Motivação e Controle.}

Fonte: Autores

Conforme apresentado na Figura 3, para as menções realizadas durante o teste, 51 respostas dos participantes foram associadas à dimensão Controle, 56 respostas associadas à dimensão Satisfação e 45 respostas associadas à dimensão Motivação.

A partir desse resultado efetuou-se uma interpretação compreensiva da informação resultante, confrontada com os dados quantitativos lenvando-se em consideração : os picos das curvas, os motivos das alterações e suas menções. A Figura 4 apresenta o gráfico com as curvas geradas pelo SAMCurve para os estados afetivos do participante 7 . Nos seis momentos avaliados, é possível identificar as variações relativas à experiência do participante durante utilização do sistema web.

Dos momentos analisados, o participante teve 2 respostas correspondentes ao momento de pico da curva (onde houve alteração da curva). A resposta do participante 7 no momento T1 (quando a dimensão Motivação apresenta uma curva ascendente):

- "O sistema veio para inovar e toda inovação nos motiva a utilização, sinto segurança ao utilizar”.

A resposta do participante 7 no momento T2 (quando a dimensão Motivação apre- 


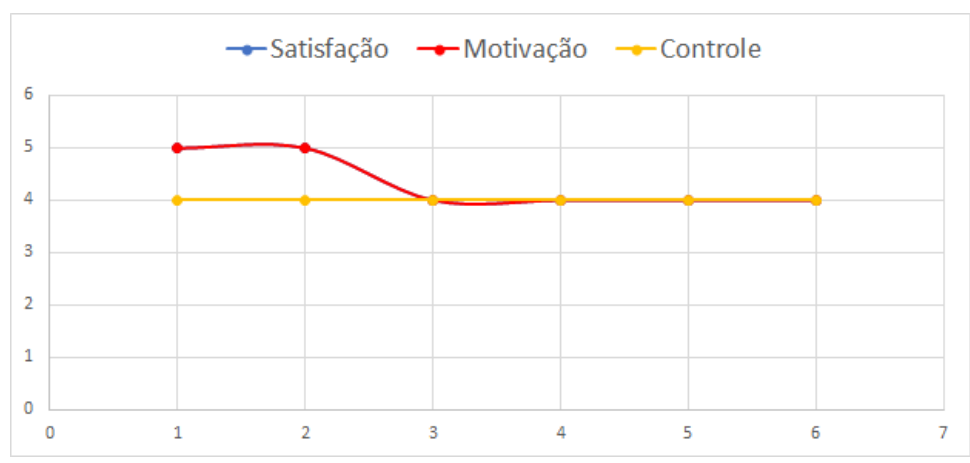

Figura 4. Representações gráficas do SAMCurve para os estados afetivos do participante 7.

Fonte: Autores

senta estabilidade no valor):

- "Recursos suficientes que nos ajudam na gestão dos controles de acesso à empresa”.

A Figura5 apresenta o gráfico com as curvas, geradas pelo SAMCurve, para os estados afetivos do participante 19. Nos seis momentos avaliados, é possível identificar as variações em relação à experiência desse participante na utilização do sistema web.

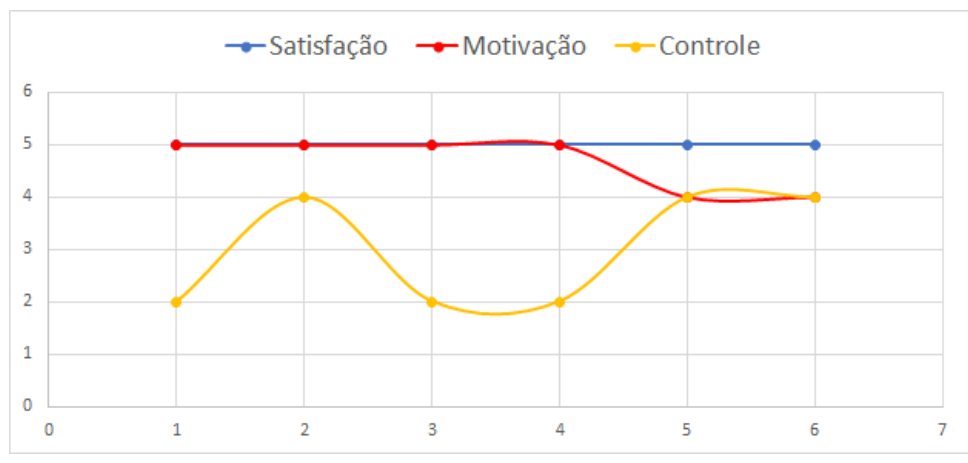

Figura 5. Representações gráficas do SAMCurve para os estados afetivos do participante 19.

Fonte: Autores

Dos momentos analisados, o participante teve 2 respostas correspondentes ao momento de pico da curva (onde houve alteração da curva). A resposta do participante 19 no momento T2 (quando a dimensão Controle apresenta uma curva ascendente):

- "Se aperfeiçoando cada dia mais".

A resposta do participante 19 no momento T3 (quando a dimensão Controle apresenta uma curva descendente):

- “Um pouco lento Porém melhorando a cada dia”.

\section{Considerações finais}

Este estudo foi longitudinal e exploratório em sua natureza, testou-se o método SAMCurve para avaliar a experiência de usuário no uso de um sistema web ao longo de um 
período predeterminado. O estudo tinha como objetivo testar a utilidade do método e sua relação com as qualidades hedônicas da experiência do usuário, visando apurar se esse método pode ser usado como uma ferramenta simples, para entender os motivos pelos quais a experiência do usuário melhora ou piora a longo prazo. Os resultados obtidos sugerem que esse método mostrou-se adequado para avaliar as qualidades hedônicas da experiência ao longo do tempo de utilização. A técnica possibilita que os usuários expressem os motivos que tornam sua experiência positiva ou negativa, além de demonstrar graficamente as alterações dos aspectos emocionais ao longo do tempo. Algumas limitações foram observadas neste estudo. Houve muitas respostas inconsistentes e repetidas, porém não impactaram nos resultados, pois foram descartados após a codificação; além disso, o sistema web avaliado pode apresentar outras dificuldades que não foram identificadas por meio da avaliação do SAMCurve feita neste estudo, pois esta avaliação foi limitada a avaliar uma atividade específica dos participantes no uso de um sistema web.

\section{Referências}

ABComm. Associação Brasileira de Comércio Eletrônico (ABComm) (2020). Crescimento do E-commerce e o Coronavírus.

ABES. Associação Brasileira das Empresas de Software (ABES) (2021). Mercado brasileiro de software: Panorama e tendências.

Alves, E. M. (2020). Sistemas computacionais tangíveis e o design da comunicação em contextos educacionais inclusivos. Tese (Doutorado em Ciência da Computação), Universidade Estadual de Campinas, Campinas.

Bradley, Margaret M e Lang, P. J. (1994). Measuring emotion: the self-assessment manikin and the semantic differential. Journal of behavior therapy and experimental psychiatry, 25(1):49-59.

BRASIL (2021). Ministério da Saúde, Conselho Nacional de Saúde - CNS. Recomendação $n^{\circ}$ 036, de 11 de Maio 2020.

Cavalcanti, I. M. F. (2020). Tecnologias em tempos de isolamento social, volume 7. RFB Editora, BELÉM-PA, $1^{\mathrm{a}}$ edition.

Forlizzi, J. and Battarbee, K. (2004). Understanding experience in interactive systems. In Proceedings of the 5th Conference on Designing Interactive Systems: Processes, Practices, Methods, and Techniques, DIS '04, page 261-268, New York, NY, USA. Association for Computing Machinery.

Kujala, S., Roto, V., Väänänen-Vainio-Mattila, K., Karapanos, E., and Sinnelä, A. (2011). Ux curve: A method for evaluating long-term user experience. Interacting with computers, 23(5):473-483.

Lazar, J., Feng, J. H., and Hochheiser, H. (2017). Research Methods in Human-Computer Interaction. Elsevier Science.

Norman, D. A. (2006). O design do dia a dia. Anfiteatro, first edition.

Teixeira, F. (2014). Introdução e boas práticas em UX Design. Casa do Código, São Paulo.

Werneck, G. L. and Carvalho, M. S. (2020). A pandemia de covid-19 no brasil: crônica de uma crise sanitária anunciada. 